\title{
Design of a Widely Tunable Modulated Grating Y-branch Laser using the Additive Vernier Effect for Improved Super-Mode Selection
}

\author{
Jan-Olof Wesström, ${ }^{\text {a }}$ Stefan Hammerfeldt, ${ }^{a}{ }^{\text {Jens Buus, }},{ }^{\mathrm{b}}$ Robert Siljan, ${ }^{\mathrm{a}}$ \\ Reinhard Laroy, ${ }^{\mathrm{c}}$ and Harry de Vries ${ }^{\mathrm{a}}$
}

\begin{abstract}
${ }^{a}$ ADC Inc. PO Box 911, SE-175 29 Jarfalla, Sweden, Tel: +46 8 50679700, Fax: +46 8 50679710, email: jan_olof_wesstrom@adc.com, b Gayton Photonics Ltd., 6 Baker Street, Gayton Nothants, NN7 3EZ, UK, Tel: $+\overline{4}$ (0)1604 859253, Fax: +44 (0)1604 859256, email: buus@compuserve.com, ${ }^{\mathrm{C}}$ Ghent University - IMEC, Department of Information Technology, St-Pietersnieuwstraat 41, B-9000 Gent, Belgium, Tel: +32-9-2643316, Fax: +32-9-2643593 , email: rlaroy@intec.rug.ac.be
\end{abstract}

\begin{abstract}
We present the design of a Modulated Grating Y-branch laser containing a splitter and two multi-peak reflectors. High selectivity is achieved using the additive Vernier effect.
\end{abstract}

Widely tunable lasers find an increasing number of applications since they can contribute to flexibility in WDM systems. ${ }^{1}$ The GCSR (Grating-assisted codirectional Coupler with Sampled Reflector) ${ }^{\text {and }}$ and SSG-DBR (Super Structure Grating Distributed Bragg Reflector) 3 lasers are two types of monolithic edge-emitting lasers based on multi-peak modulated reflector gratings such as the SSG or the sampled grating. Also the Modulated Grating Y-branch lasert(MGY) presented here (Figure 1 ) is based on multi-peak reflectors and can be designed to cover the entire C-band $(192-196 \mathrm{THz})$. In our design the light is split by the use of a $60-\mu \mathrm{m}$ long, $7-\mu \mathrm{m}$ wide Multi-Mode

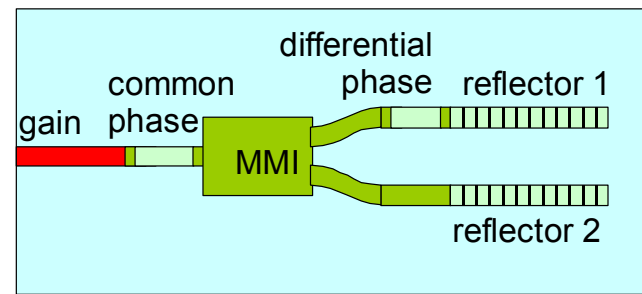

Figure 1. Schematic drawing of the MGY Interferometer (MMI). Then 46- $\mu \mathrm{m}$ long S-bends with a $130-\mu \mathrm{m}$ radius of curvature are used to increase the separation between the waveguides. Each arm ends with a 500- $\mu \mathrm{m}$ long multi-peak reflector with an effective coupling coefficient of $30 / \mathrm{cm}$. In one arm there is an $80-\mu \mathrm{m}$ long phase section that can be used to adjust the phase difference between the reflections. A common phase section of the same length is used to align the cavity mode with the reflector peaks. The design is to be manufactured as an InP/InGaAsP buried hetero structure, butting a $400 \mu \mathrm{m}$ multi-quantum-well gain section to the passive sections made in a $0.35-\mu \mathrm{m}$ thick InGaAsP layer with a photo-luminescence wavelength of $1.39 \mu \mathrm{m}$.

The MGY is similar to the SSG-DBR in that it is based on the Vernier effect using two multi-peak reflection gratings. The SSG-DBR utilizes two such gratings, one at each end of the cavity. The reflection peak separations of the two gratings are slightly different and the Vernier effect is used to select one reflection peak for lasing. By tuning the two reflection sections and a phase section, any frequency within a wide range can be reached. One disadvantage with the SSG-DBR laser is that the output light has to pass through the front reflector, which inevitably suffers from free carrier absorption when tuned. This gives a higher power variation. The MGY is also comparable to the GCSR laser in the sense that it has all tuning sections on the same side of the gain section, so that the power can exit the cavity without absorption. Instead of using the Vernier effect, the GCSR laser uses a widely tunable coupler transmission peak to efficiently select one peak for lasing. One disadvantage for the GCSR is that it is typically a rather long chip.
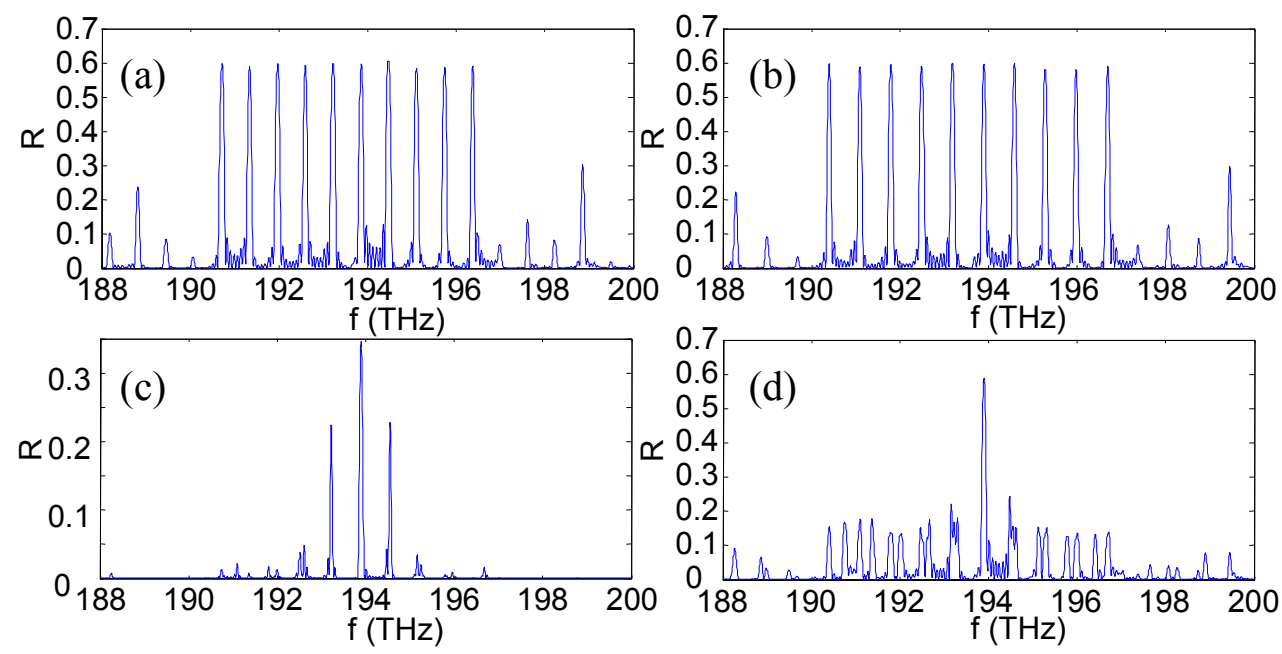

Figure 2. Power reflection spectra of (a) reflector 1 and (b) reflector 2, with 630 and $700 \mathrm{GHz}$ peak spacing respectively. (c) The product of the spectra, $\left|r_{1} r_{2}\right|^{2}$, after a slight tuning of reflector 1 so that peaks 6 are aligned (SSG-DBR case). (d) Sum of the reflections, $\left|r_{1}+r_{2}\right|^{2} / 4$, (MGY case). 
Conceptually, one main difference between the SSG-DBR laser and the MGY laser is that, for the MGY, the super-mode selection is performed by an addition of the complex amplitudes of reflectivities instead of a multiplication. Since the addition is sensitive to the phase difference between the reflections, the shape of the aggregate reflection spectrum is not self-evident. As seen in Figure 2 (d), when properly designed the addition suppresses the adjacent peaks strongly although these side peaks overlap. The large overlap is evident from the product of the two reflections seen in Figure 2 (c). The MGYlaser has an extra differential phase section that controls the phase difference between the two reflections. It is highly desirable to eliminate the need to readjust this setting between different operation points. Also this can be done by following a set of simple design rules.

It can easily be shown that if the reflections are to stay in phase without changing the differential phase section, while the two reflectors are simultaneously tuned by the same amount (medium tuning) the reflector tuning contacts have to start at the same distance from the splitter. When making a super-mode hop from peaks $m$ to peaks $m+1$, (coarse tuning) a further requirement for the phase conservation is that $\phi_{2}{ }^{m+1}-\phi_{1}{ }^{m+1}=\phi_{2}{ }^{m}-\phi_{1}{ }^{m}$, where $\phi_{i}^{m}$ is the phase before tuning at reflection peak $m$ in reflector $i$ using the beginning of the tuning contacts as reference points. Some frequencies can most easily be reached using peak $m$ in reflector 1 and peak $m+1$ in reflector 2 or vice versa (repeat mode tuning). For those operation points to work properly we also require that $\phi_{2}{ }^{m+1}=\phi_{2}{ }^{m}$ and $\phi_{1}{ }^{m+1}=\phi_{1}{ }^{m}$. If this is to hold for all peaks, their phases would all have to be the same which is only possible for the simple sampled reflector, which has the disadvantage that the reflection amplitude varies among the peaks. Fortunately frequency coverage can be

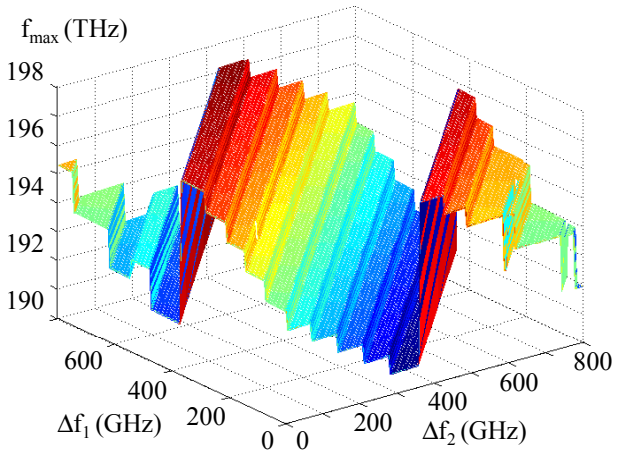

Figure 3. Peak frequency for the aggregate reflection in the MGY case. achieved without using repeat mode tuning for all peaks, at the cost of slightly higher tuning currents. As mentioned the suppression of reflection peaks adjacent to the ones that currently overlap is very sensitive to the phase. It can be shown that the phase difference, $\Psi$, at which these peaks add is $\Psi=4 \pi n_{g l} L_{e f f}\left(\Delta f_{2}-\Delta f_{1}\right) / c_{0}$, where $L_{\text {eff }}$ is the average effective length of the reflectors counted from the beginning of the contacts, $\Delta f_{i}$ is the peak separation in reflector $i$, and $n_{g r}$ is the group index in both reflectors. The expression shows that, just as in the SSG-DBR case, reflector pairs with high effective coupling constant (low $L_{\text {eff }}$ ) or a small difference in peak separation will have less suppression of adjacent peaks. The example in Figure 2 however shows that the selectivity is much higher for the MGY case, at the cost of low suppression of peaks further away. These peaks may cause problems only when the gain spectrum is not sufficiently flat over the intended tuning range.

After designing the reflectors according to these design rules, a simulation was performed showing that all frequencies between 191.335 and $196.77 \mathrm{THz}$ can be reached by tuning each reflector by less than $800 \mathrm{GHz}$ without readjusting the differential phase section. See Figure 3. For the whole frequency range, the main aggregate reflection peak was at least 1.9 times larger (in power reflection) than the second largest one. Since the differential phase section can be set to the same value for all operation points, it is tempting to take it away to shorten the laser. This is indeed possible although it requires careful control in manufacturing. Simulations show that a variation of \pm 1 radian in phase difference can be accepted with maintained frequency coverage. This corresponds to a difference in effective index of the waveguides of the order of \pm 0.01 or a difference in the overall positioning of the reflector gratings of $+-35 \mathrm{~nm}$, clearly achievable with electron-beam lithography. The elimination of the $4^{\text {th }}$ tuning section reduces the control complexity to the level of an SSG-DBR. A further reduction in length can be achieved by letting the MMI together with the bends act as the common phase section. Then the length would be very close to a 3-section DBR laser achieving a larger tuning range although lower tuning currents are used.

Due to the free carrier absorption, reflectivities are reduced with tuning. For the design described in the introduction, the simulations show that the effective reflection, as seen from the gain section, falls in the range between $10.5 \%$ and $17.8 \%$ for all operation points in the covered tuning range. Eliminating the differential phase section and letting the MMI and the bends double as a phase section, the effective reflectivities fall in the range between $15.6 \%$ and $26.4 \%$. These are higher numbers than what can easily be achieved with the GCSR. Assuming a gain current of $150 \mathrm{~mA}$ and a front facet reflectivity of $28 \%$, the output power is estimated to vary between $13 \mathrm{~mW}$ and $16 \mathrm{~mW}$, for the shortened design. Applying an AR coating of $5 \%$ is estimated to boost the output power to values between $25 \mathrm{~mW}$ and $28 \mathrm{~mW}$.

The MGY is currently under production and further progress will be reported at the conference. This work has been supported by the EU via the IST project IST-2000-2844 NEWTON.

\footnotetext{
${ }^{1}$ R. Plastow, "Emerging tunable laser applications in optical networks, "Lightwave Magazine, vol 17, no. 3, March 2000.

2 J. Willems, G. Morthier, and R. Baets, "Novel widely tunable integrated optical filter with high spectral selectivity," Proc. ECOC '92, pp. 413-416, paper WeB9.2, Berlin, Germany, September 1992.

${ }^{3}$ V. Jayaraman, Z.-M. Chuang, and L.A. Coldren, "Theory, design and performance of extended tuning range semiconductor lasers with sampled gratings," IEEE J. Quantum Electron., vol. 29, no. 6, pp. 1824-1834, June 1993.

${ }^{4}$ G Sarlet, J Buus, R Baets, European patent application EP 99870214
} 